\title{
Béatrice Bloch, Une lecture sensorielle : le récit contemporain, Gracq, Simon, Kateb, Delaume
}

Christine Genin

\section{OpenEdition}

1 Journals

Édition électronique

URL : http://journals.openedition.org/ccs/1840

DOI : $10.4000 /$ ccs. 1840

ISSN : 2558-782X

Éditeur :

Presses universitaires de Rennes, Association des lecteurs de Claude Simon

Édition imprimée

Date de publication : 30 août 2018

Pagination : 214-218

ISBN : 978-2-7535-7489-2

ISSN : 1774-9425

Référence électronique

Christine Genin, «Béatrice Bloch, Une lecture sensorielle : le récit contemporain, Gracq, Simon, Kateb, Delaume », Cahiers Claude Simon [En ligne], 13 | 2018, mis en ligne le 30 août 2019, consulté le 24 septembre 2020. URL : http://journals.openedition.org/ccs/1840; DOI : https://doi.org/10.4000/ccs. 1840 
Béatrice Bloch, Une lecture sensorielle : le récit contemporain, Gracq, Simon, Kateb, Delaume, PUR, coll. «Interférences ", 2017, 211 p.

Béatrice Bloch, au fil des études qu'elle nous propose, est à la recherche de ce qui fait le plaisir de la lecture. Après une thèse intitulée «Inscription et rôle du lecteur dans l'espace romanesque contemporain ", qui s'efforçait, en 1994, de démontrer qu' "une expérience émotionnelle n'a lieu que lorsque le texte ne sature pas l'imaginaire ", elle a publié en 1998 un premier essai, Le Roman contemporain: liberté et plaisir du lecteur ${ }^{2}$ où il était déjà question de Claude Simon et où elle se demandait: "Qu'est-ce qui fait une expérience de lecture réussie? Est-ce la liberté laissée au lecteur d'interpréter le texte à sa guise? Est-ce au contraire la sensation de se trouver emporté par le récit à un endroit imprévu? Est-ce encore le plaisir d'imaginer? "Son postulat était déjà que l'émotion du lecteur est suscitée par la lettre du texte, c'est-à-dire à la fois par ses images et par son tempo, mais que l'imagination de l'interprète ne peut se mettre en ouvre que si une certaine disponibilité intellectuelle lui est laissée. À cette seule condition peut naître une affinité entre le texte et le moi du lecteur.

En 2013, elle a également publié un article sur " La construction de l'émotion chez le lecteur: Immersion et persuasion esthétique ${ }^{3} »$ : « La fiction ou la littérature, ce n’est évidemment pas la vie, mais une ou des figurations d'univers, mimétiques ou non, de l'univers réel. Comment, dès lors, imaginer que l'émotion puisse être ressentie et les lecteurs s'alarmer, s'amuser et prendre part au texte avec émotion? » Elle y transposait une description de la manière dont un acteur entre dans un rôle à la description de la lecture: le lecteur entre dans une quasi-émotion et se trouve dans un état d'esprit comparable à celui qui serait le sien face à une véritable expérience, tout en gardant la conscience que l'expérience n'est pas réelle. C'est ce que Jean-Marie Schaeffer, dans Pourquoi la fiction?, appelle la "feintise ludique ${ }^{4} »$.

Dans le présent essai, Béatrice Bloch recentre son propos sur le récit en prose poétique, en précisant que cette expression désigne «tout texte en prose, qu'il se réclame de l'imaginaire ou non, et qui joue sur une poéticité de sa texture, c'est-à-dire ralentit la lecture en travaillant particulièrement images, rythmes, choix de narration, modes d'énonciation" (p. 7). Elle choisit comme exemples de récit en prose poétique des textes de Julien Gracq, Claude Simon, Yacine Kateb et Chloé Delaume, les auteurs cités dans le titre, mais aussi de bien d'autres écrivains, assez divers: Alain Robbe-Grillet, Olivier Cadiot, H.P. Lovecraft, Marguerite Duras, François Bon, Nathalie Sarraute, Patrick Chamoiseau, André du Bouchet ou Stéphane Mallarmé notamment.

Béatrice Bloch se demande alors comment un lecteur parvient à entrer dans un récit, lorsque celui-ci est écrit en prose poétique et de ce fait résiste à l'adhésion fic-

2. Le Roman contemporain: liberté et plaisir du lecteur (sur Butor, Des Forêts, Sarraute, Pinget), L'Harmattan, coll. «Critiques littéraires », 1998.

3. "La construction de l'émotion chez le lecteur: Immersion et persuasion esthétique ", Poétique, $\mathrm{n}^{\circ} 163$, 2013, p. 339-348, [https://www.cairn.info/revue-poetique-2010-3-page-339.htm].

4. J.-M. Schaeffer, Pourquoi la fiction?, Le Seuil, coll. « Poétique », 1999. 
tionnelle? Elle entend ainsi proposer une théorie de l'expérience mentale, imaginaire et sensorielle du lecteur. L'hypothèse mise à l'épreuve est que la lecture de textes en prose poétique permet une expérience sensorielle feinte, produisant plaisirs et sensations, comme si le lecteur avait un " corps fictif»:

Nous tenterons ici de prouver que le lecteur se trouve doté d'un corps-esprit imaginaire, expliquant à la fois qu'il soit capable de percevoir et de comprendre ce qui est écrit, de s'y immerger, et d'éprouver du plaisir à le lire, comme à le déguster. Et ce corps fictif est créé par les stratégies d'écriture, qui proposent comme autant de personnalités diverses que le lecteur endosse, le temps de sa lecture. (p. 8)

Sont particulièrement intéressantes ses analyses concernant la thymie, qui à la différence de la passion ou de la phorie, indique le côté passager d'une disposition mentale particulière: les «aspects thymiques pris dans le texte ont un impact sur le lecteur dont ils déterminent une certaine disposition d'un corps et d'un esprit fictifs, dispositions d'humeur vues par le lecteur [...] qui participent de la mise en place d'une expérience sensorielle feinte » (p. 16).

Dans une première partie, Béatrice Bloch analyse les conditions d'entrée dans la lecture, et tente de définir l'aptitude imaginaire comme pouvoir de projection et de figuration en convoquant Freud, Kant, Sartre, Damasio et Schaeffer. Comment le lecteur entre-t-il dans un récit ou une fiction caractérisés par une prose poétique? Quelles sont les conditions du fonctionnement imaginaire qui lui permettent de s'immerger dans les textes? C'est la capacité humaine à se promener dans un univers fictif grâce à l'imagination et aux constructions linguistiques proximisantes qui actualisent les descriptions qui permettent d'associer des sensations évoquées dans un texte à des sensations semblables mémorisées par le lecteur. Elle passe en revue les définitions de ce qu'est l'imaginaire, comme capacité à s'immerger ou à s'impliquer dans ce qui est lu: "capacité à la promenade d'un corps fictif " grâce aux embrayeurs et aux temps du récit, capacité de synthèse, grâce à l'existence de procédures permettant de revivifier la coordination de perceptions sensorielles, de se souvenir et de lire et surtout penchant naturel vers l'affectivité (plaisir ou déplaisir) : "La synthèse opérée par le texte replie le divers du réel et permet au lecteur, a posteriori, de déplier alors, sous l'encodage réduit, la totalité d'une expérience » (p. 198).

Dans la seconde partie, Béatrice Bloch fait l'inventaire des artefacts soumis au lecteur: images, sons, rythmes. Elle formule l'hypothèse qu'il existe deux types de vécus différents dans la lecture: une expérience de perception sensorielle directe (sons prononcés intérieurement, dessins des lettres sur la page) et une expérience oblique, vécue par décodage et mentalisation, la représentation intérieure des images. Quels sont les divers modes d'immersion dans un texte? En premier lieu "l'immersion par les sons et les rythmes, artefacts vécus de la lecture " (p. 145). Le lecteur peut reconnaître des scénarios et des expériences vécues ou leur transposition, mais il existe également un vécu propre à la sphère esthétique: la récurrence de fragments élémentaires (les yx et or de Mallarmé), de thématiques et de noyaux imaginaires récurrents, crée un mode 
spécifique, que le lecteur apprivoise au cours de la lecture. En second lieu « l'immersion par les images, artefacts sémantisés et mentalisés» (p. 198): Béatrice Bloch opère une distinction entre les artefacts vécus ou directs de la lecture et les artefacts " obliques » demandant une représentation mentale ou " mentalisation ». En troisième lieu, la réception des figures par des lecteurs réels. Elle se livre à une expérience de réception de l'hypotypose à partir d'extraits de La Jalousie d'Alain Robbe-Grillet, avant de conclure que « les images signifiantes sont des tropes ou des symboles, les images sensorielles sont décrites avec tant de détails qu'on a l'impression de faire, grâce à elles, de quasi-expériences, et les images mimétiques renvoient à une expérience déjà vécue par le lecteur» (p. 199).

La troisième et dernière partie est consacrée à des études expérimentales sur la combinaison des expériences de réception visuelle, mentale et auditive du texte. Des textes de Chloé Delaume, de Lovecraft, de Claude Simon et de Julien Gracq sont proposés à des lecteurs et les résultats d'enquêtes sont présentés. Les textes d'André du Bouchet et de Chloé Delaume démontrent une "compatibilité expérimentale des expériences de réception musicale et de réception figurale ». Les textes de Lovecraft, Julien Gracq et Claude Simon sont l'occasion d'analyser "la réception imaginaire: sensorialités et émotions, ensemble ".

S’agissant de Claude Simon, Béatrice Bloch avait déjà publié «Vers une sensorialité pure de la lecture? Visualisation d'une lecture de La Bataille de Pharsale de Claude Simon ". Dans la deuxième section du chapitre 5 de la troisième partie (p. 165-187), intitulée "Proposition pour la visualisation d'une expérience de lecture: l'expérience psychique et l'expérience sensorielle combinée " elle parvient, à partir d'un extrait de La Bataille de Pharsale, à bien montrer l'enchevêtrement des différents états du lecteur face au texte simonien.

Partant du principe qu'il lui semble nécessaire de figurer une expérience de lecture, à la prime découverte du texte, en tentant de séparer les diverses actions du lecteur en réponse aux grains textuels variés, elle visualise sous la forme de schémas (ressemblant un peu à des électrocardiogrammes ou à des partitions pour orchestre) les actions/réactions du lecteur, réparties entre différentes lignes (thymique/psychique/rythmique/des images/sensorielle/d'expérience imaginaire) qui prennent à tour de rôle le dessus dans la partition de la lecture.

Outre celle de la partition musicale, Béatrice Bloch utilise également, pour décrire la lecture, l'image du parfumeur qui compose un parfum:

Le lecteur entre dans le texte par l'expérience psycho-affective, ou par les jeux esthétiques. Comme le parfumeur, il joue des différentes fragrances séparément ou ensemble, crée une symphonie à partir de ce que le texte lui donne, se laisse prendre par les images ou par les rythmes, se projette dans des émotions temporaires, ou au contraire a des réticences devant une texture qui le déborde ou lui déplaît et qu'il rejette (p. 195).

5. "Vers une sensorialité pure de la lecture? Visualisation d'une lecture de La Bataille de Pharsale de Claude Simon ", Cahiers de Narratologie, n 11, 2004, consulté le 29 juillet 2017, [http://narratologie.revues.org/7]. 
Elle parvient en cela fort bien me semble-t-il à montrer «ce qui se passe de manière latente dans sa riche lecture ordinaire d'un récit en prose poétique " (ibid.).

Au-delà de ce chapitre entièrement consacré à La Bataille de Pharsale, Béatrice Bloch a aussi évoqué plus haut d'autres romans de Clause Simon à l'appui de ses développements: la description des graffitis, dans La Bataille de Pharsale encore, lui semble renvoyer le lecteur vers l'enfance et la "délectation sensorielle devant l'opacité d'un monde dont nous ne pouvons comprendre le sens ", associée à la "richesse pulsatile " des rythmes et des longues phrases (p. 87-90). Dans la mise en page du Jardin des Plantes, elle voit une "traduction du temps mental ", la fragmentation du texte renvoyant paradoxalement à une " unité de conscience " qui est celle du souvenir, dont la tension ou l'émotion traverse la fragmentation de surface (p. 110-112). Elle s'interroge aussi brièvement sur la manière dont le lecteur s'approprie et reçoit émotionnellement la "boue gluante et maternelle" de La Route des Flandres (p. 118).

La description du concert dans Histoire est également analysée très finement comme un exemple d'hypotypose réussie: "l'immersion donne à vivre l'expérience elle-même, comme en direct [...] le vécu du lecteur est alors immersif, ses perceptions visuelles étant plongées dans la situation fictive" (p. 121-122). Béatrice Bloch se penche également avec bonheur sur la description de New York dans Le Jardin des Plantes (p. 127129), description fascinante car " elle est sous le signe de la décomposition du réel en ses constituants élémentaires [...] dotant la ville de New York d'une vie de gemmeplante ". Le caractère anti-intuitif et la bizarrerie de l'image lui confèrent paradoxalement un caractère "sidérant " et l'image " ébahit le lecteur ":

La force de Claude Simon ici, est de faire tenir ensemble des sensations visuelles élémentaires [...] avec une connaissance figurée de New York. Cette métaphore-métamorphose est lisible car elle contient des éléments tirés de sensations connues [...] sur lesquelles nous pouvons dès lors greffer notre propre imaginaire (p. 128).

Béatrice Bloch parvient dans cet ouvrage à bien mettre en évidence l'éventail des actions et réactions du lecteur, selon le grain textuel auquel il est confronté, qui font le " caractère combinatoire de la lecture " (p. 26). Cela lui permet d'approcher le mystère de ce qui fait que «la lecture offre l'accès à une réalité augmentée » (p. 26). Elle conclut très joliment:

Il nous est apparu que la lecture mettait en œuvre des expériences réelles et mentales où s'apprivoisent l'innovation et l'inouii. La lecture, en prose poétique, parce qu'elle plonge dans un univers à visée totale, joue à la fois sur des sensorialités et sur l'immersion, la simulation. [...] c'est la puissance esthétique du texte qui, mimesis mise à part, offre des vécus intenses comme autant d'incitations à aller vers un texte parfois rétif ou mystérieux. Sensations et émerveillement s'y allient; ils produisent alors le plaisir de la simulation réussie d'expérience. (p. 201)

On pourrait formuler un petit bémol: les conclusions de Béatrice Bloch ne sont pas toujours totalement convaincantes car son propos, qui se veut scientifique et fait régulièrement état des "expériences " menées auprès de lecteurs pour étayer des hypothèses, gagnerait peut-être à emprunter davantage aux neurologues ou neurobiologistes. On a en 
effet souvent l'impression de raisonnements qui s'arrêtent en chemin ou tournent court car il leur manque une explication liée au fonctionnement du cerveau ou de la conscience.

Mais l'ensemble du volume offre néanmoins une intéressante promenade, ponctuée d'analyses très précises et stimulantes de textes eux-mêmes passionnants, ceux des auteurs cités dans le titre mais aussi bien d'autres, de Duras à Mallarmé. Le lecteur en ressort avec une conscience encore un peu plus nette de la complexité extrême de la pratique de la lecture littéraire, et plus encore de ce qui fait qu'elle est un plaisir. On est encore assez loin d'avoir totalement défini la lecture, mais c'est probablement une très bonne chose.

\section{Christine Genin}

\section{Mireille Calle-Gruber, Agnès Cousin de Ravel et François Migeot (dir.), Présences de Claude Simon, Mont-de-Laval, L'Atelier du Grand Tétras, 2016, 213 p.}

Le volume réunit les interventions proposées lors des Journées culturelles Claude Simon qui se sont tenues à Arbois, en octobre 2013, pour le centenaire de la naissance du romancier. Sur la couverture figure une photographie montrant Simon et Peter Brugger aux Planches en 1975 pendant le tournage du court-métrage L'Impasse. Réalisé à partir de Triptyque, le film, à sa manière, porte "les souvenirs du Jura " dont le romancier affirme qu'ils "sont ce qu'il a de plus cher ${ }^{6}$ ". Présences de Claude Simon est sous-tendu par la tension qui travaille l'œuvre de Simon et qui en reçoit ici une acuité plus vive d'être animée de la puissance de l'émotion: qu'en est-il des attachements personnels dans un roman soucieux des formes de ses constructions?

Sous l'intitulé "Claude Simon, le goût des mots et des formes », M. Calle-Gruber, frappe les premières notes des "Introductions " pour donner la mesure de ce qu'est Arbois pour Simon: le lieu d'affinités électives autant que d'attaches affectives tissées tout au long d'une vie et tramées dans le corps de l'œuvre. Dans le second temps introductif, François Migeot examine la «Permanence de Claude Simon »: si la fidélité au pays arboisien est vécue et écrite, elle crée sa forme propre qui ne relève ni du sentimentalisme régionaliste, ni de la servilité au réel de l'autobiographie.

Un ensemble de contributions, "Atelier du roman : le devenir-texte ", mène dès lors sur le terrain complexe des transformations romanesques de la matière d'Arbois.

Alastair B. Duncan délivre un " petit historique " de l'édition des œuvres de Simon dans la bibliothèque de la Pléiade. Maître d'œuvre de l'aventure éditoriale commencée sous la houlette du romancier, il décline avec précision et humour les raisons de l'ensemble: contraintes, justification de la distribution en deux volumes, défis à relever par les différents contributeurs (information, choix d'une approche critique).

6. C. Simon, lettre à Alice Mathiot du 19 janvier 1978, p. 204. Dans cette lettre, il déclare également souhaiter être « enterré dans la tombe des Simon, dans le petit cimetière près du confluent des deux rivières » (p. 205). 